\title{
Dark energy and dark matter interaction in light of the second law of thermodynamics
}

\author{
Víctor H. Cárdenas ${ }^{1, \mathrm{a}}$, Daniela Grandón ${ }^{1, \mathrm{~b}}$, Samuel Lepe $\mathrm{e}^{2, \mathrm{c}}$ \\ ${ }^{1}$ Instituto de Física y Astronomía, Universidad de Valparaíso, Gran Bretaña 1111, Valparaíso, Chile \\ ${ }^{2}$ Instituto de Física, Pontificia Universidad Católica de Valparaíso, Av. Brasil 2950, Valparaíso, Chile
}

Received: 6 February 2019 / Accepted: 16 April 2019 / Published online: 24 April 2019

(C) The Author(s) 2019

\begin{abstract}
In the context of thermodynamics we discuss the way inevitable emerge an interaction between dark components, and in this way, provide a mechanism to understand the limits of the LCDM model and the class of interaction models between dark components. Using observational data we have tested two particular models of explicit interaction between dark components and reconstructed the evolution of temperatures for both components. We found that observations suggest the interaction exist with energy flowing from dark energy to dark matter. The best fit also suggest a phantom equation of state parameter for dark energy. We discuss the results having in mind the constraints imposed by thermodynamics.
\end{abstract}

\section{Introduction}

One of the main problems in cosmology today is to identify or characterize the cause of the accelerated expansion found in $[1,2]$, usually called dark energy (DE). Although there have been, from time to time, evidence for DE evolution, or in its primordial form, evidence for a variable cosmological constant, the case have taken new impulse after the results using the BAO BOSS DR11 [3]. In that work, by interpreting the results in a simple $\omega \mathrm{CDM}$ model, strongly suggest a $2.5 \sigma$ departure from $\Lambda \mathrm{CDM}$ at $z=2.34$. Soon after this work appears, several papers focused on finding what model better describe the results from the BOSS analysis. In [4] the authors found evidence for DE evolution using the $H(z)$ measurement implied by [3]. They propose a model where the cosmological constant was screened in the past. Also in [5] the authors demonstrate that it is possible to explain the BOSS result in the context of the interacting dark scenario

\footnotetext{
a e-mail: victor.cardenas@uv.cl

b e-mail:dgrandons@gmail.com

${ }^{c}$ e-mail: samuel.lepe @ pucv.cl
}

(dark matter (DM) interacting with DE), excluding null interaction at $2 \sigma$. Also in [6] the authors found similar results to [4]. Regarding the dark interaction scenario, using data from Planck, type Ia supernova, and redshift space distortions, in [7] the authors found statistical evidence for a dark interacting model (the so called Interacting vacuum model, named iVCDM, [8]) starting at $z \simeq 0.9$.

One of the main problems with dark interaction models is the arbitrariness in the coupling, usually named $Q$. In particular, in the iVCDM the coupling is written as $Q=-q_{v} H \rho_{v}$, where $H$ is the Hubble function, $q_{v}(z)$ is the arbitrary constant usually parameterized in bins to be reconstructed by observations, and $\rho_{v}$ is the vacuum energy density. In some sense, the same pathological feature the cosmological constant $\Lambda$ has in the $\Lambda$ CDM model, here emerges through $Q$ in the interacting dark matter/energy model. So it would be interesting to find ways to constraint $Q$ not only through observations but also from physical principles.

In this paper, we want to explore to what extent, the interaction term $Q$ can be determined and constrained by appealing to thermodynamic considerations. In what follows we use natural units, $c=8 \pi G=k_{B}=1$. The paper is organized as follows: in the next section we discuss the thermodynamics in the context of an interacting model. In Sect. 3 we discuss the method of effective equation of state and obtain the expressions for the temperature as a function of redshift. Then we describe the two particular models of interaction we study and the data we have used to constraint the models in Sect. 5 . We end with the discussion of our results.

\section{Thermodynamics and interaction}

\subsection{A single fluid}

Let us start with general considerations about thermodynamics in an expanding Universe using a single fluid. Assuming 
a flat universe and a homogeneous component we get the Hubble equation and the conservation equation

$3 H^{2}=\rho, \quad \dot{\rho}+3 H(\rho+p)=0$,

where $H=\dot{a} / a$ is the Hubble parameter and $a(t)$ the scale factor. From the second law of thermodynamics applied to a comoving volume element of unit coordinate volume (and physical volume $V=a^{3}$ ) we can write

$T d S=d[(\rho+p) V]-V d p$.

Here $\rho$ is the energy density, $p$ is the pressure, $T$ the temperature of the system and $S$ the total entropy per comoving volume. Using the fact that $S$ is an state function we get

$\frac{\rho+p}{T}=\frac{d p}{d T}$,

then Eq. (2) can be written as

$d S=\frac{d[(\rho+p) V]}{T}-V(\rho+p) \frac{d T}{T^{2}}=d\left[\frac{(\rho+p) V}{T}\right]$,

which means that entropy can be written as

$S=$ const. $+\frac{(\rho+p) a^{3}}{T}$.

On the other hand, from Einstein's equations we get the energy conservation Eq. (1) that can be written as

$d[(\rho+p) V]=V d p$

which means that the entropy (5) is constant during the expansion,

$S=\frac{(\rho+p) a^{3}}{T}=$ const.

This results emphasize the adiabatic expansion universe picture. Then Einstein's equations implies adiabatic expansion assuming $T \neq 0$. Also, Eq. (7) tell us that the special combination of physical variables keep a constant value once the evolution of $p(a), \rho(a)$ and $T(a)$ are introduced. Clearly, once we know the equation of state (EoS), we can infer the temperature evolution of the system.

Another way to see this, is by using that our single component satisfies number and energy conservation. Following [10] it is possible to find the relation

$\dot{T}=-3 H T \frac{\partial p}{\partial \rho}$.
This equation also enable us to find how the temperature evolves if an EoS is given. For example, for $p=\omega \rho$ we get

$T(a) \simeq a^{-3 \omega}$,

that gives the expected answer for radiation $\left(\omega_{r}=1 / 3\right)$. The same result can be obtained from Eq. (7). In general, assuming $\omega=\omega(a)$ we can write

$T(a)=T\left(a_{0}\right) \exp \left(-3 \int_{a_{0}}^{a} d a \frac{\omega(a)}{a}\right)$.

\subsection{Two fluids}

Let us assume now two fluids - DM and DE for example and assume that both components conserved separately, i.e. they satisfy

$$
\begin{array}{r}
\dot{\rho}_{x}+3 H\left(1+\omega_{x}\right) \rho_{x}=0, \\
\dot{\rho}_{m}+3 H\left(1+\omega_{m}\right) \rho_{m}=0,
\end{array}
$$

so no interaction is present. For each component is possible to write the same equations derived before. In particular, we can write the evolution of temperature for these two components as

$$
T_{m} \simeq \frac{1}{a^{2}}, \quad T_{x} \simeq \exp \left(-3 \int d a \frac{\omega_{x}}{a}\right),
$$

where we have used the previous result for dust. It is clear that this result suggest that today $T_{m} \ll T_{x}$ because $\omega_{x}<0$, as was first notice in [9]. It is also clear that in this case the expansion is adiabatic, because each one of the conservation equations above can be written as $d U+p d V=0$ where $U=\Omega_{v} \rho a^{3}$ and $V=\Omega_{v} a^{3}$ where $\Omega_{v}$ is a volume constant factor.

The current observational evidence - so far - support the $\Lambda \mathrm{CDM}$ model, where $\Lambda$ is a component that can be considered as a fluid with EoS parameter $\omega=-1$, plus a cold (non relativistic velocities) DM component, both of which conserved separately, i.e., they do not interact. In this context we can ask, what temperature can be associated to this $\Lambda$ fluid? From our previous considerations, assuming $p=\omega \rho$ with $\omega=$ const. we can write

$d S=(1+\omega) d\left(\frac{\rho V}{T}\right)$,

from which we can obtain

$(1+\omega) \rho V=$ const. $\times T$.

This relation suggest that for a fluid with $\omega=-1$ we get $T=0$, which according to the third law of thermodynamics implies $S=0$. This result - independent of the awkward 
features of existence of a pure cosmological constant component - tells us that such a component is at least inconsistent with thermodynamic too.

Moreover, for the case $\omega<-1$ ? i.e., the phantom case, following the same argument we get $T<0$, and if we use the Euler relation

$T S=(1+\omega) \rho V \Longrightarrow T S<0 \rightarrow S>0$,

which is the well known phantom problem. In this regard in [11] a solution is proposed in the context of irreversible thermodynamics.

\subsection{Two fluids interacting}

The concordance cosmological model assumes that every component - baryons, radiation, DM and DE - conserve separately. Then as we have discussed in the previous section, the universe expands adiabatically. Because both dark components can only be detected through their gravitational effects, is not easy to distinguish their effects and in principle they can interacts between them while the total energy density of the dark sector is conserved. Phenomenologically this can be described by the conservation equations as

$$
\begin{gathered}
\dot{\rho}_{x}+3 H\left(1+\omega_{x}\right) \rho_{x}=-Q, \\
\dot{\rho}_{m}+3 H\left(1+\omega_{m}\right) \rho_{m}=Q .
\end{gathered}
$$

According to (2) these equations tell us that the entropy for each component is not constant. By re-written each one as the first law we get for the interaction function $Q$

$Q=-\frac{T_{x}}{V} \frac{d S_{x}}{d t}=\frac{T_{m}}{V} \frac{d S_{m}}{d t}$.

No interaction $Q=0$ means both $S_{m}$ and $S_{x}$ being constants, i.e. we have an adiabatic evolution, in contrast to an interacting model, where a $Q \neq 0$ leads to

$T_{m} d S_{m}+T_{x} d S_{x}=0$

then we get non-adiabaticity. By using (19) we can also see this in another form by written

$$
\begin{aligned}
d\left(S_{m}+S_{x}\right)= & -\left(\frac{T_{m}}{T_{x}}-1\right) d S_{m} \neq 0 \Longrightarrow S_{m} \\
& +S_{x} \neq \text { const. }
\end{aligned}
$$

According to (19) also we get that

$$
Q>0 \Longrightarrow d S_{x}<0 \text { and } d S_{m}>0
$$

implying the DE entropy decreases as the DM entropy increases. From (20) it is direct to write

$\frac{d}{d t}\left(S_{x}+S_{m}\right)=-\left(\frac{T_{m}}{T_{x}}-1\right) \frac{d S_{m}}{d t} \gtrless 0 \Longrightarrow T_{m} \lessgtr T_{x}$,

because $d S_{m} / d t>0$. Then at any instant DM is cooler than the DE. We can also write this last relation using (19) to get

$$
\begin{aligned}
\frac{d}{d t}\left(S_{x}+S_{m}\right) & =\left(\frac{1}{T_{m}}-\frac{1}{T_{x}}\right) V Q \\
& >0 \Longleftrightarrow Q>0 \text { and } T_{m}<T_{x} .
\end{aligned}
$$

This means that, if we require that the second law be valid in this case, then we have to have $T_{m}<T_{x}$. This is an interesting result, because we do not have any idea of the magnitudes of these temperatures. There are attempts to determine the case for $T_{m}$ using candidates particles for DM, but we do not have any idea about $T_{x}$ for DE. This is also interesting to notice that this result, that $T_{m}<T_{x}$ is also in connection with the inequality $Q>0$, making clear the relation between the validity of the second law with a positive $Q$ which means a transfer of energy from DE to DM.

Having found these results, let us discuss a specific model. Let us study an interacting model close to the $\triangle \mathrm{CDM}$ one. According to (17) and (18)

$\dot{\rho}_{x}+3 H\left(1+\omega_{x}\right) \rho_{x}=-Q=\left(\frac{1}{V}\right) T_{x} \frac{d S_{x}}{d t}$,

$\dot{\rho}_{m}+3 H\left(1+\omega_{m}\right) \rho_{m}=Q=\left(\frac{1}{V}\right) T_{m} \frac{d S_{m}}{d t}$,

and for the purpose in hands, let us assume $\omega_{x}=-1(\Lambda)$, and $\omega_{m}=0(C D M)$, then we get

$\dot{\rho}_{x}=-Q \Longrightarrow \rho_{x}=-\int d t Q$,

$\dot{\rho}_{m}+3 H \rho_{m}=Q$,

which reduces to $\Lambda \mathrm{CDM}$ for $Q=0$ zero interaction. This model is actually the $\mathrm{i} \Lambda \mathrm{CDM}$ model of [8]. It was this model that was tested in [7] against observation finding positive evidence for interaction. Given there are evidence for an evolving $\rho_{x}$ that means - given the well known results in [12] we have also evidence for an interacting model.

For a component $\rho_{x}=\Lambda\left(\omega_{x}=-1\right)$ it is clear that $Q=0$ and then $T_{m} d S_{m} / d t=0$, and beacuse $T_{m}=$ const., then $S_{m}=$ const. And also as we saw before, because $T_{x}\left(\omega_{x}=-1\right)=0$ and $S_{x}\left(\omega_{x}=-1\right)=0$.

In conclusion, $\Lambda C D M$ is well supported by observations and also the assumption of adiabatic evolution ( $S=$ const.) is consistent with the philosophy of the standard model that ensures adiabatic evolution after inflation ends. Then - if there is evidence for an interaction between DE and DM - 
then in general the universe evolution is no adiabatic. Thus, our considerations on thermodynamics implies that a pure $\Lambda$ component is hardly consistent with physics, unless we accept that both components - DM and DE - interact with each other.

\section{Effective temperature method (ETM)}

In this section we study the thermal evolution in an interaction DE/DM model using an effective equation of state. We follow previous works $[13,14]$ on the subject. Let us write the system $(25,26)$ as

$\dot{\rho}_{x}+3 H\left(1+\omega_{x}^{e f f}\right) \rho_{x}=0$,
$\dot{\rho}_{m}+3 H\left(1+\omega_{m}^{e f f}\right) \rho_{m}=0$,

where

$\omega_{x}^{e f f}=\omega_{x}+\frac{Q}{3 H \rho_{x}} \quad$ and $\quad \omega_{m}^{e f f}=\omega_{m}-\frac{Q}{3 H \rho_{m}}$,

then according to (10) we can write

$T(z)=T(0) \exp \left(3 \int_{0}^{z} \frac{d z}{1+z} \omega^{e f f}(z)\right)$.

where we have used that $1+z=a_{0} / a$, and so for each dark component from (17) and (18) we can write

$T_{x}(z)=T_{x}(0)(1+z)^{3 \omega_{x}} \exp \left[\int_{0}^{z} d \ln (1+z)\left(\frac{Q}{H \rho_{x}}\right)\right]$,

$T_{m}(z)=T_{m}(0)(1+z)^{3 \omega_{m}} \exp \left[-\int_{0}^{z} d \ln (1+z)\left(\frac{Q}{H \rho_{m}}\right)\right]$,

and from this, we see that if interaction exists for $\omega_{m}=0$ (CDM) we get $T_{m}(z) \neq$ const.

Let us apply this result to the most well known Ansatzes: $Q=3 \gamma H \rho_{x}$ and $Q=3 \gamma H \rho_{m}$. These ansatzes can be considered as a good probe to explore small departures from the usual evolution of each component. For example, in the absence of interaction in a flat universe the DM component evolves as $a^{-3}$. A small departure from this behavior can be parameterized as $a^{-3(1+\beta)}$ with $\beta$ small. In this case, according to Eq. (18) an interaction term of the type $\alpha 3 H \beta \rho_{m}$ is suggested. Then, under the assumption that these interaction are small - which is supported by the success of the $\Lambda \mathrm{CDM}$ model - we proceeds studying these two specific models.
3.1 Ansatz $Q=3 \gamma H \rho_{x}$

In this case, we get

$$
\begin{aligned}
T_{x}(z) & =T_{x}(0)(1+z)^{3\left(\omega_{x}+\gamma\right)} \\
T_{m}(z) & =T_{m}(0)(1+z)^{3 \omega_{m}} \exp \left[-3 \gamma \int_{0}^{z} d \ln (1+z)\left\{\frac{1}{r(z)}\right\}\right] .
\end{aligned}
$$

where $r(z)=\rho_{m}(z) / \rho_{x}(z)$ is the coincidence parameter. In order to get a close solution for both temperatures we need the solution for both energy densities first. In this case - using the Eq. (29) - we get the solution

$\rho_{x}(z)=\rho_{x}(0)(1+z)^{3\left(1+\omega_{x}+\gamma\right)}$.

Using this last result in the conservation equation for dark matter $\rho_{m}$ in Eq. (30) and using that $x=a / a_{0}$ and $d / d t \rightarrow$ $d / d a$, we can write the equation as

$\frac{d \rho_{m}}{d x}+\frac{3\left(1+\omega_{m}\right)}{x} \rho_{m}=3 \gamma \rho_{x}\left(a_{0}\right) x^{-\left[3\left(1+\omega_{x}+\gamma\right)+1\right]}$,

whose solution is given by

$$
\begin{aligned}
\rho_{m}(x)= & \exp \left[-\frac{3\left(1+\omega_{m}\right)}{x}\right]\left[C_{2}+3 \gamma \rho_{x}\left(a_{0}\right)\right. \\
& \left.\times \int d x \exp \left[\frac{3\left(1+\omega_{m}\right)}{x}\right] x^{-\left[3\left(1+\omega_{x}+\gamma\right)+1\right]}\right]
\end{aligned}
$$

The integral can be done directly, recalling that $x=a / a_{0}=$ $(1+z)^{-1}$. In this way we get $\rho_{m}(z)$ and consequently $r(z)$ y $T_{m}(z)$, according to (36).

\subsection{Ansatz $Q=3 \gamma H \rho_{m}$}

Using this ansatz, with $\gamma>0$ we get

$T_{x}(z)=T_{x}(0)(1+z)^{3 \omega_{x}} \exp \left[3 \gamma \int_{0}^{z} d \ln (1+z) r(z)\right]$,

$T_{m}(z)=T_{m}(0)(1+z)^{3\left(\omega_{m}-\gamma\right)}$,

Again, from the conservation Eq. (30) we get

$\rho_{m}(z)=\rho_{m}(0)(1+z)^{3\left(1+\omega_{m}-\gamma\right)}$

Using this in the conservation equation for $\rho_{x}$ we get

$\dot{\rho}_{x}+3 H\left(1+\omega_{x}\right) \rho_{x}=-3 \gamma H \rho_{m}\left(a_{0}\right)\left(a_{0} / a\right)^{3\left(1+\omega_{m}-\gamma\right)}$. 
Following the previous case we get

$$
\begin{gathered}
\rho_{x}(x)=\exp \left[-\frac{3\left(1+\omega_{x}\right)}{x}\right]\left[C_{1}-3 \lambda_{m} \rho_{m}\left(a_{0}\right)\right. \\
\left.\int d x \exp \left[\frac{3\left(1+\omega_{x}\right)}{x}\right] x^{-\left[3\left(1+\omega_{m}-\lambda_{m}\right)+1\right]}\right]
\end{gathered}
$$

and the integral can be done explicitly. In this way knowing $\rho_{x}(z)$ and then $r(z)$ and $T_{x}(z)$ according to (40).

\section{The models}

In this section we write explicitly the formulae to be used in the study of restrictions imposed by observational data. Here we study the two models of interaction discussed in the previous section, $Q_{1}=3 \gamma H \rho_{x}$, and $Q_{2}=3 \gamma H \rho_{m}$. Both have been already studied in [15]. If $\gamma$ is zero, then there is no interaction.

Let us start with the set of equations for the dark components $(17,18)$. We assume here that the EoS parameter for $\mathrm{DM}$ is $\omega_{m}=0$, and we let free the EoS parameter for DE $\omega_{x}$.

$\dot{\rho}_{x}+3 H\left(1+\omega_{x}\right) \rho_{x}=-Q$,

$\dot{\rho}_{m}+3 H \rho_{m}=Q$.

For the first model (i), we can solve (45) for $\rho_{x}$ first. We find that $\rho_{x} a^{3\left(1+\omega_{x}+\gamma\right)}=$ const. Then we replace this in (46) to find an explicit form for $\rho_{m}(a)$. Having both energy densities we can insert these in the Hubble equation

$H^{2}=\kappa\left(\rho_{m}+\rho_{m}+\rho_{r}\right)$

where $\kappa=8 \pi G / 3$ and we have assumed a flat universe. Writing everything in terms of the redshift $1+z=a_{0} / a$, the Hubble function $H(z) / H_{0}=E(z)$ for model (i) is given by

$$
\begin{aligned}
E^{2}(z)= & \Omega_{m}(1+z)^{3}+\Omega_{r}(1+z)^{4} \\
& +\Omega_{x}\left(\frac{\gamma}{w+\gamma}(1+z)^{3}+\frac{w}{w+\gamma}(1+z)^{3(1+w+\gamma)}\right),
\end{aligned}
$$

where $\Omega_{r}=2.469 \times 10^{-5} h^{-2}\left(1+0.2271 N_{\text {eff }}\right)$ and $N_{\text {eff }}=$ 3.04 , and $\gamma$ is the parameter that makes the interaction manifest. Here $\Omega_{m}=\Omega_{c}+\Omega_{b}$, where $\Omega_{c}$ is the non-baryonic part and $\Omega_{b}$ is the baryonic one.

For the second model (ii), we obtain

$$
\begin{aligned}
E^{2}(z)= & \Omega_{x}(1+z)^{3(1+w)}+\Omega_{r}(1+z)^{4}+\Omega_{b}(1+z)^{3} \\
& +\Omega_{c}\left(\frac{\gamma}{w+\gamma}(1+z)^{3(1+w)}+\frac{w}{w+\gamma}(1+z)^{3(1-\gamma)}\right) .
\end{aligned}
$$

Here the free parameters are $h, \Omega_{b}, \Omega_{c}, w$ and $\gamma$. It is clear that for $\gamma=0$ both expressions - those for models (i) and (ii) - reduced to that of the $\omega \mathrm{CDM}$ model.

\section{The data}

In this section we describe the five sets of observational data we have used to put constraints on the models we have defined in the previous section. Preliminary results of this work appears in [16]. We use: measurements of the Hubble function $H(z)$, from type Ia supernova (SNIa), baryonic acoustic oscillations (BAO), gas mass fraction in clusters $f_{\text {gas }}$ and from Cosmic Microwave Background Radiation (CMBR).

The 31 data points for the Hubble function $H(z)$ are taken from several works and were compiled by [17] expanding a redshift range from $z=0.07$ to $z=1.965$. In summary it comprise data points from [18-20] and also from [21]. It is important to notice that we have used only those $H(z)$ measurements obtained using the differential age method [22], and we have explicitly exclude those obtained using the clustering method, because we are also using data from BAO.

The latest sample of SNIa is the Pantheon sample [23]. Here we use the chi square function defined as

$\chi^{2}=\left(\mu-\mu_{t h}\right)^{T} C^{-1}\left(\mu-\mu_{t h}\right)$,

where $C$ corresponds to the covariance matrix delivered in [23], $\mu_{t h}=5 \log _{10}\left(d_{L}(z) / 10 p c\right)$ is the distance modulus where $d_{L}(z)$ is the luminosity distance, and the modular distance is assumed to take the shape

$\mu=m-M+\alpha X-\gamma Y$,

where $m$ is the maximum apparent magnitude in band $\mathrm{B}, X$ is related to the widening of the light curves, and $Y$ corrects the color. The cosmological parameters are then constrained along with the parameters $M, X$ and $Y$. Also from [23] a binned version of the data was published where only $M$ is a free parameter.

The data points we use for BAO are those compiled in [24]. This set comprise data from the 6dF survey [25] at redshift $z=0.106$, distance measurements from [26] at redshift $z=0.15$, and with data from the Baryon Oscillation Spectroscopic Survey (BOSS) at redshifts $z=0.32, z=0.57$ and $z=2.34$. In all these cases the baryonic peak is estimated performing an average in the radial and transverse direction. At higher redshift it is possible to measure the BAO scale in the radial and tangential directions simultaneously, proving measurements of the Hubble parameter $H(z)$ and the angular diameter distance $D_{A}(z)$. 
The BAO observations give information about the ratio

$$
\frac{D_{A}(z)}{r_{s}}=\frac{P}{(1+z) \sqrt{-\Omega_{k}}} \sin \left(\sqrt{-\Omega_{k}} \int_{0}^{z} \frac{d z}{E(z)}\right),
$$

for the transverse direction. Here $P=c /\left(r_{s} H_{0}\right)$ and it takes the value $30.0 \pm 0.4$ for the best $\Lambda \mathrm{CDM}$ Planck fit, $r_{s}$ is the co-moving sound horizon that according to Planck it takes the value $r_{s}=1059.68$ [27], and also information about the ratio

$\frac{D_{H}(z)}{r_{S}}=\frac{P}{E(z)}$,

for the line-of-sight direction. The parameter $P$ was used in [24] to perform an unanchored BAO analysis, which does not use a value for $r_{s}$ obtained from a cosmological constant, also performed in [28].

At low redshift, because it is not possible to disentangle the BAO scale in the transverse and radial direction, the surveys give the value for the ratio $D_{V}(z) / r_{s}$, where

$D_{V}(z)=\left[z(1+z)^{2} D_{A}(z)^{2} D_{H}(z)\right]^{1 / 3}$,

which is an angle-weighted average of $D_{A}$ and $D_{H}$. From [24] the data considered are: at low redshift, at $z=$ 0.106 we have $D_{V} / r_{s}=2.98 \pm 0.13$, and for $z=$ $0.15, D_{V} / r_{s}=4.47 \pm 0.17$. For high redshift we consider $0.00874 D_{H} / r_{s}+0.146 D_{A} / r_{s}=1.201 \pm 0.021$ and $0.0388 D_{H} / r_{s}-0.0330 D_{H} / r_{s}=0.781 \pm 0.053$ at $z=$ $0.32 ; 0.0158 D_{H} / r_{s}+0.101 D_{A} / r_{s}=1.276 \pm 0.011$ and $0.0433 D_{H} / r_{s}-0.0368 D_{H} / r_{s}=0.546 \pm 0.026$ at $z=0.57$. Following [24], in order to use the BAO measurements for the Lyman $\alpha$, we used the $\chi^{2}$ files supplied on the website [29] directly. In what follows, we take the Planck value for $r_{s}$ and use $P$ as a function of $H_{0}$.

Data from measurements of gas mass fraction in clusters, $f_{\text {gas }}$ was also used assuming they are sources of X-ray as suggested by [30]. In particular we use the data from [31] which consist in 42 measurements of the X-ray gas mass fraction $f_{\text {gas }}$ in relaxed galaxy clusters in the redshift range $0.05<z<1.1$. To determine constraints on cosmological parameters we use the model function [32]

$f_{\text {gas }}^{\Lambda C D M}(z)=\frac{b \Omega_{b}}{(1+0.19 \sqrt{h}) \Omega_{M}}\left[\frac{d_{A}^{\Lambda C D M}(z)}{d_{A}(z)}\right]^{3 / 2}$,

where $d_{A}(z)$ is the angular diameter distance, $b$ is a bias factor which accounts that the baryon fraction is slightly lower than for the universe as a whole. From [33] it is obtained $b=$ $0.824 \pm 0.0033$. In the analysis we also use standard priors on $\Omega_{b} h^{2}=0.02226 \pm 0.0023$ and $h=0.678 \pm 0.009$ [34].
We also use CMB data in the form of the acoustic scale $l_{A}$, the shift parameter $R$, and the decoupling redshift $z_{*}$. The $\chi^{2}$ for the CMB data is constructed as

$\chi_{C M B}^{2}=X^{T} C_{C M B}^{-1} X$,

where

$X=\left(\begin{array}{c}l_{A}-302.40 \\ R-1.7246 \\ z_{*}-1090.88\end{array}\right)$.

The acoustic scale is defined as

$l_{A}=\frac{\pi r\left(z_{*}\right)}{r_{S}\left(z_{*}\right)}$,

and the redshift of decoupling $z_{*}$ is given by [35],

$z_{*}=1048\left[1+0.00124\left(\Omega_{b} h^{2}\right)^{-0.738}\right]\left[1+g_{1}\left(\Omega_{m} h^{2}\right)^{g_{2}}\right]$,

$g_{1}=\frac{0.0783\left(\Omega_{b} h^{2}\right)^{-0.238}}{1+39.5\left(\Omega_{b} h^{2}\right)^{0.763}}$,
$g_{2}=\frac{0.560}{1+21.1\left(\Omega_{b} h^{2}\right)^{1.81}}$,

The shift parameter $R$ is defined as in [36]

$R=\frac{\sqrt{\Omega_{m}}}{c\left(1+z_{*}\right)} D_{L}(z)$.

$C_{C M B}^{-1}$ in Eq. (56) is the inverse covariance matrix,

$C_{C M B}^{-1}=\left(\begin{array}{ccc}3.182 & 18.253 & -1.429 \\ 18.253 & 11887.879 & -193.808 \\ -1.429 & -193.808 & 4.556\end{array}\right)$.

More details of the work with the data see [37].

\section{Results}

For the analysis we have used the code EMCEE [38]. It is a Python module that implement an Affine-invariant Markov chain Monte Carlo (MCMC) method. We have perform the analysis using the five data sets mentioned in the previous section. In practice we have considered a burn-in phase where we monitoring the auto-correlation time $(\tau)$ and set a target number of independent samples. Then, we set 10000 MCMC steps $(\mathrm{N})$ with a number of walkers in the range between 50 and 100. Our estimations of the auto-correlation times for each parameter in the three models all satisfies the relation $N / \tau \gg 50$ suggested in [38], a condition that is considered a good measure of assets convergence in our samplings. 
Table 1 Best fit values of the cosmological parameters for the interaction models using $\mathrm{SNIa}+H(z)+\mathrm{BAO}+f_{\text {gas }}+\mathrm{CMB}$

\begin{tabular}{lcc}
\hline & $Q=3 H \gamma \rho_{m}$ & $Q=3 H \gamma \rho_{x}$ \\
\hline$h$ & $0.669 \pm 0.008$ & $0.671 \pm 0.009$ \\
$\Omega_{c}$ & $0.301 \pm 0.004$ & $0.300 \pm 0.004$ \\
$\Omega_{b}$ & $0.049 \pm 0.001$ & $0.048 \pm 0.001$ \\
$\omega$ & $-1.03 \pm 0.02$ & $-1.05 \pm 0.02$ \\
$\gamma$ & $0.071 \pm 0.006$ & $0.07 \pm 0.005$ \\
\hline
\end{tabular}

The results are shown in Table 1, and the best fit plots are shown in Fig. 1 for model (i) and in Fig. 4 for model (ii).

As can be seen, the best fit with the full set of observational data in both models indicates positive evidence for interaction - with a similar value for $\gamma \simeq 0.07$ - and that the transfer of energy flows from DE to DM. Based on the considerations we have made in previous sections, we find that our theoretical constraints are in good agreement with the observational evidence. We also notice that the EoS parameter for DE $\omega$ is less than -1 in both cases, pointing towards evidence for phantom dark energy. The rest of the parameters take best fit values that are not too different from the usual ones. The results for the best fit for model (i) is shown in Fig. 1.

Using these best fit values, we can use Eqs. (35 and 36) to reconstruct the temperatures for DE and DM. In Fig. 2 we show the result for model (i).

According to what we expect, the temperature of the dark energy grows with the expansion, and through the interac-

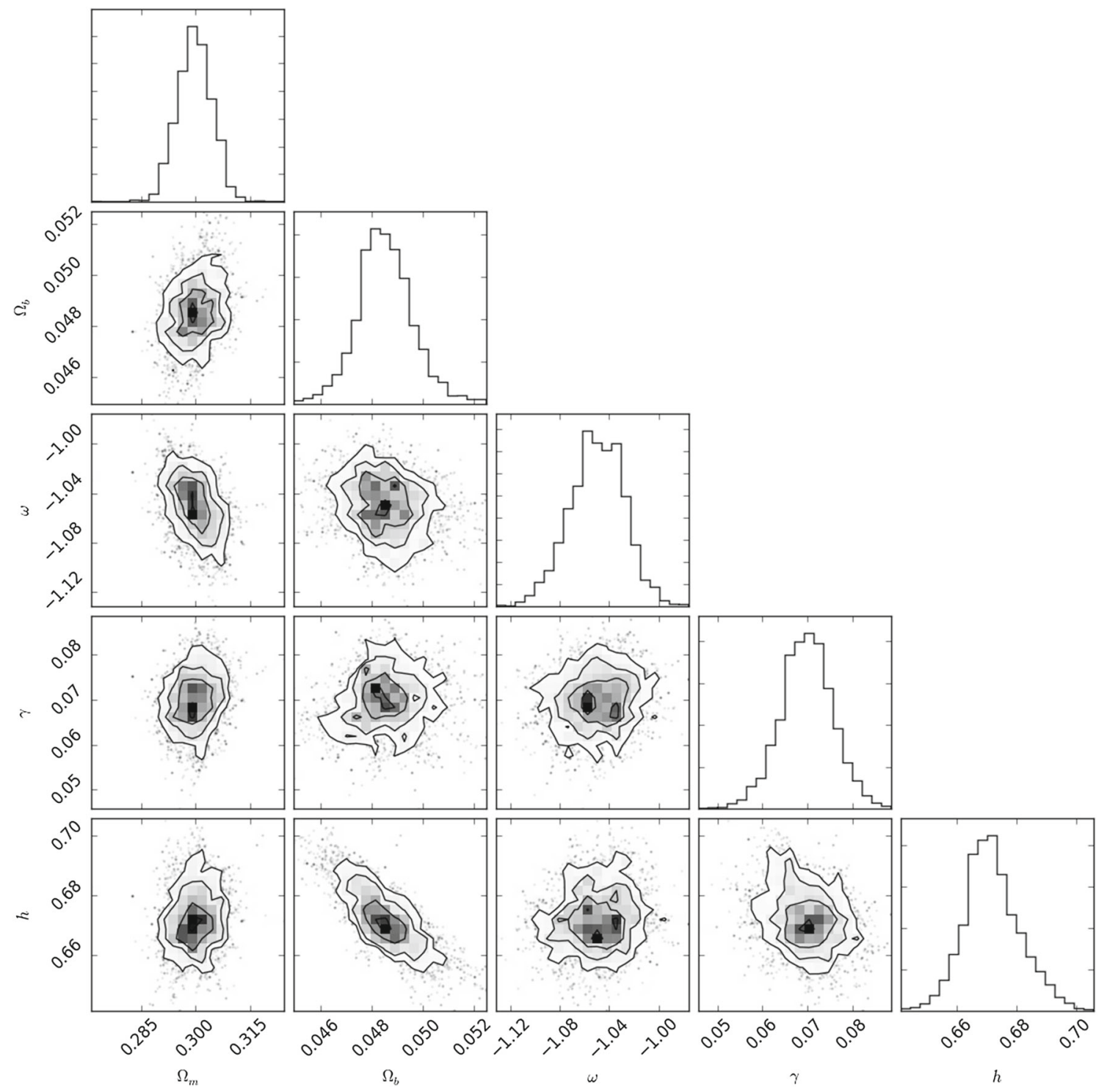

Fig. 1 We display the results for $1 \sigma, 2 \sigma$ and $3 \sigma$ for the model (i) in the parameter space $\left(\Omega_{m}, \Omega_{b}, \omega, \gamma, h\right)$ using all the data 


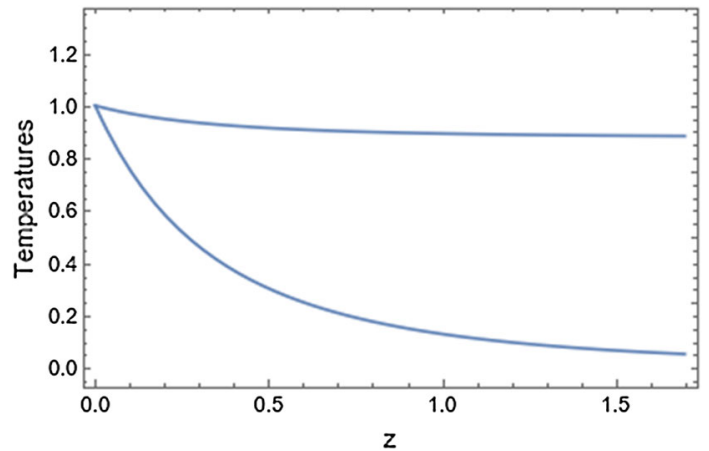

Fig. 2 We display the reconstructed temperatures for model (i) using the expressions $(35,36)$ using all the data. The upper line is $T_{m}(z) / T_{m}(0)$ and the lower one is $T_{x}(z) / T_{x}(0)$

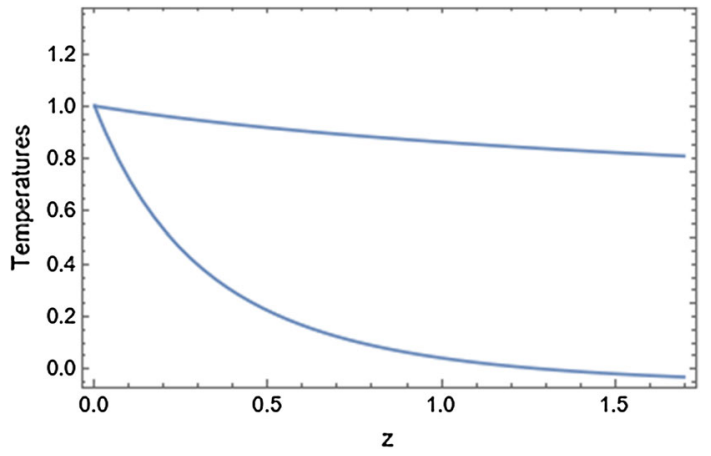

Fig. 3 We display the reconstructed temperatures for model (ii) using the expressions (40) and (41) using all the data. The upper line is $T_{m}(z) / T_{m}(0)$ and the lower one is $T_{x}(z) / T_{x}(0)$

tion, causes also that the temperature of dark matter to grow as well, much more moderately, but it increases. This is expected because, without interaction, DM temperature is constant, so once there is a transfer of energy from DE to $\mathrm{DM}$, it will result in an increase in temperature, since the DE temperature increases with the expansion.

In the case of model (ii) the results are very similar as can be seen in Table 1. The best fit values of the parameters are shown in Fig. 4. The reconstructed temperatures are also very similar as can be seen in Fig. 3.

In order to determine how good our interacting models are in describing the data, we use two well know statistic tools: the Akaike Information Criteria (AIC) based on information theory and the Bayesian Information Criteria (BIC) based on Bayesian inference [39]. In Table 2 we display the results of the analysis using the definition for AIC and BIC.

According to these results, between our reference model $(\omega \mathrm{CDM})$ and the interacting model (i) we find $\triangle \mathrm{AIC}=2$ indicating that both models are as good as each other describing the data. However, using the $\triangle \mathrm{BIC}=4.82$ indicates a positive evidence against the fit of model (i). This is not a surprise, the BIC generally penalizes free parameters more
Table 2 Comparison among the models under study. The non interacting $\omega \mathrm{CDM}$, and the interacting models, (i) $Q_{1}=3 H \gamma \rho_{m}$ and model (ii) $Q_{2}=3 H \gamma \rho_{x}$. We display the $\chi_{\min }^{2}$ value for the best fit using all the observational probes, together the value for the AIC and BIC as is discussed in the text

\begin{tabular}{llll}
\hline & $\omega \mathrm{CDM}$ & $Q_{1}$ & $Q_{2}$ \\
\hline$\chi_{\min }^{2}$ & 134.85 & 134.85 & 127.63 \\
AIC & 142.85 & 144.85 & 137.63 \\
BIC & 154.13 & 158.95 & 151.73 \\
\hline
\end{tabular}

strongly than the Akaike information criterion. In the case of model (ii) we get $\triangle \mathrm{AIC}=5.2$ and $\triangle \mathrm{BIC}=2.4$, indicating that this model is as good as the reference model in describing the data.

In this section we have performed an analysis using five geometric probes to constrain two interacting models. Compared to previous analysis as in [15], we have used more data probes but we have obtained higher values for $\gamma$ in both cases. The reason behind this finding could be the use of BAO data at larger redshift as those at $z=2.34$ and $z=2.36$ not used in [15] and also the use of gas mass fraction data. We know that the inclusion of the BAO data points suggest strongly a departure from $\Lambda \mathrm{CDM}$ and a preference for an interaction model [3-5,37]. It could be also the use of $f_{\text {gas }}$ data, a set with well known tension with $\Lambda \mathrm{CDM}[40]$. According to the analysis by using both information and bayesian criteria, model (ii) is far better than model (i) in describing the data, but however although the AIC criteria does not exclude the possibility that model (i) can describe the data as good as the model $\omega \mathrm{CDM}$, the BIC criteria suggest that model (ii) is not better than the reference model. In the case of model (ii) compared to the reference model, we find that both criteria suggest that model (ii) performs better than the $\omega \mathrm{CDM}$ (Fig. 4).

Although we have performed an analysis in the context of an interacting model, it is interesting to come back to the thermodynamic features of the $\Lambda \mathrm{CDM}$ model. As we have discussed here, a pure $\Lambda$ component - understood as a source in the right hand side of Einstein's equations - does not have any sense thermodynamically. In fact, as we have found in Sect. 2, the entropy associated to $\Lambda$ should be zero during all the universe evolution. This implies that a more sound model is necessary to explain what we are observing. Assuming that the EoS parameter evolve with redshift is equivalent to consider an interaction between components, in our case DE and DM, so, a more physical model - thinking in thermodynamics - must consider an interaction, something that the observational data seems to support.

Finally, it is very difficult to measure the temperature for dark matter (see for example [41,42]). What we know from the thermodynamic considerations is that $T_{m}<T_{x}$. Certainly, more work is needed to understand the implications of the thermodynamic evolution of our universe. 


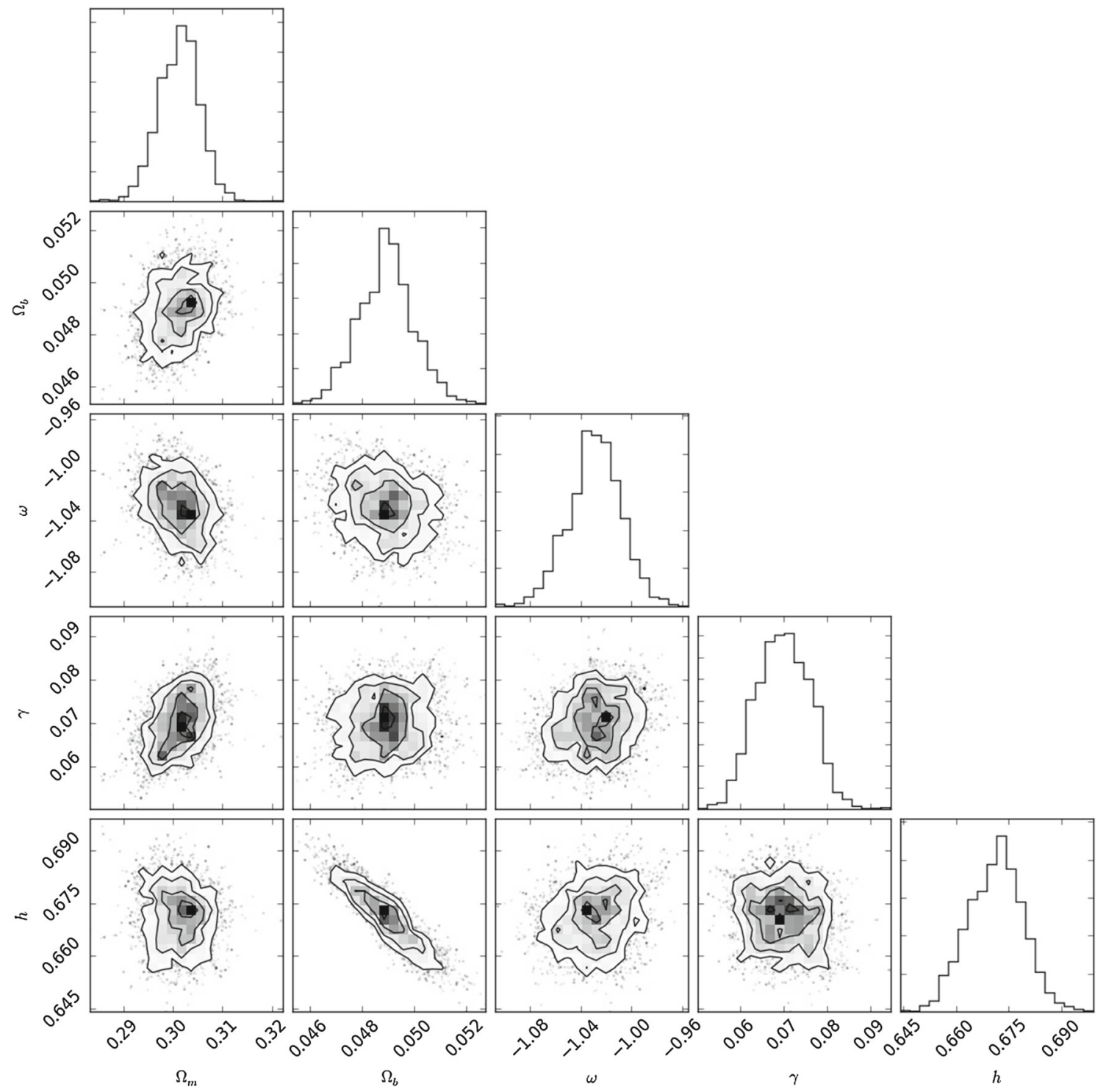

Fig. 4 We display confidence boundaries for $1 \sigma, 2 \sigma$ and $3 \sigma$ for the model (ii) for the free parameters $\left(\Omega_{m}, \Omega_{b}, \omega, \gamma, h\right)$ using all the data

Data Availability Statement This manuscript has no associated data or the data will not be deposited. [Authors' comment: All the data used in this work can be found in other works, to which we have made explicit reference in the text.]

Open Access This article is distributed under the terms of the Creative Commons Attribution 4.0 International License (http://creativecomm ons.org/licenses/by/4.0/), which permits unrestricted use, distribution, and reproduction in any medium, provided you give appropriate credit to the original author(s) and the source, provide a link to the Creative Commons license, and indicate if changes were made. Funded by SCOAP $^{3}$.

\section{References}

1. A.G. Riess et al., Astron. J. 116, 1009 (1998). arXiv:astro-ph/9805201
2. S. Perlmutter et al., Astrophys. J. 517, 565 (1999). arXiv:astro-ph/9812133

3. T. Delubac et al., BOSS Collaboration. A\&A 574, A59 (2015). arXiv:1404.1801 [astro-ph.CO]

4. V. Sahni, A. Shafieloo, A.A. Starobinsky, Astrophys. J. 793(2), L40 (2014). arXiv:1406.2209 [astro-ph.CO]

5. E.G.M. Ferreira, J. Quintin, A.A. Costa, E. Abdalla, B. Wang, Evidence for interacting dark energy from BOSS. Phys. Rev. D 95(4), 043520 (2017). arXiv: 1412.2777 [astro-ph.CO]

6. X. Ding, M. Biesiada, S. Cao, Z. Li, Z.H. Zhu, Astrophys. J. 803(2), L22 (2015). arXiv:1503.04923 [astro-ph.CO]

7. V. Salvatelli, N. Said, M. Bruni, A. Melchiorri, D. Wands, Phys. Rev. Lett. 113(18), 181301 (2014). arXiv: 1406.7297 [astro-ph.CO]

8. D. Wands, J. De-Santiago, Y. Wang, Class. Quant. Gravit. 29, $145017(2012)$

9. D. Pavon, B. Wang, Gen. Relat. Gravit. 41, 1 (2009). arXiv:0712.0565 [gr-qc]

10. R. Maartens, arxiv:astro-ph/9609119

11. M. Cruz, S. Lepe, S.D. Odintsov, Phys. Rev. D 98, 083515 (2018) 
12. Astrophys. Space Sci. Proc. 45, 323-329 (2016)

13. M. Cruz, S. Lepe, F. Peña, Phys. Rev. D 92, 123511 (2015)

14. S. Lepe, F. Peña, Eur. Phys. J. C 76(9), 507 (2016)

15. D. Xia, S. Wang, MNRAS 463, 952-956 (2016)

16. D. Grandon, V.H. Cardenas,. arXiv:1804.03296 [astro-ph.CO]

17. J. Magana, M.H. Amante, M.A. Garcia-Aspeitia, V. Motta, Mon. Not. R. Astron. Soc. 476, 1036 (2018)

18. C. Zhang et al., Res. Astron. Astrophys. 14, 1221 (2014)

19. D. Stern, R. Jimenez, L. Verde, M. Kamionkowski, S.A. Stanford, JCAP 1002, 008 (2010)

20. M. Moresco et al., JCAP 1208, 006 (2012)

21. M. Moresco, Mon. Not. R. Astron. Soc. 450, L16 (2015)

22. R. Jimenez, A. Loeb, Astrophys. J. 573, 37 (2002)

23. D.M. Scolnic et al., https://doi.org/10.17909/T95Q4X arXiv:1710.00845 [astro-ph.CO]

24. J. Evslin, JCAP 1704, 024 (2017). arXiv:1604.02809 [astroph.CO]

25. F. Beutler et al., MNRAS 416, 3017 (2011)

26. A.J. Ross, L. Samushia, C. Howlett, W.J. Percival, A. Burden, M. Manera, Mon. Not. R. Astron. Soc. 449(1), 835 (2015). arXiv:1409.3242 [astro-ph.CO]

27. P. Collaboration, Astron. Astrophys. 594, A13 (2016)

28. E. Aubourg et al., Phys. Rev. D 92, 123516 (2015)

29. http://darkmatter.ps.uci.edu/baofit/

30. S. Sasaki, Publ. Astron. Soc. Jap. 48, L119 (1996)
31. S.W. Allen, D.A. Rapetti, R.W. Schmidt, H. Ebeling, G. Morris, A.C. Fabian, Mon. Not. R. Astron. Soc. 383, 879 (2008). arXiv:0706.0033 [astro-ph]

32. S.W. Allen, R.W. Schmidt, H. Ebeling, A.C. Fabian, L. van Speybroeck, Mon. Not. R. Astron. Soc. 353, 457 (2004). arXiv:astro-ph/0405340

33. V.R. Eke, J.F. Navarro, C.S. Frenk, Astrophys. J. 503, 569 (1998). arXiv:astro-ph/9708070

34. M. Tanabashi et al., Particle Data Group. Phys. Rev. D 98, 030001 (2018)

35. W. Hu, N. Sugiyama, Astrophys. J. 471, 542 (1996)

36. J.R. Bond, G. Efstathiou, M. Tegmark, Mon. Not. R. Astron. Soc. 291, L33 (1997)

37. V.H. Cardenas, Phys. Lett. B 750, 128 (2015)

38. D. Foreman-Mackey, D.W. Hogg, D. Lang, J. Goodman, Publ. Astron. Soc. Pacific 125, 306 (2013)

39. A.R. Liddle, Mon. Not. R. Astron. Soc. 377, L74 (2007). arXiv:astro-ph/0701113

40. V.H. Cardenas, C. Bernal, A. Bonilla, Mon. Not. R. Astron. Soc. 433, 3534 (2013). https://doi.org/10.1093/mnras/stt983. arXiv:1306.0779 [astro-ph.CO]

41. D. Obreschkow, C. Power, M. Bruderer, C. Bonvin, Astrophys. J. 762, 115 (2013)

42. N. Mirabolfathi, arXiv: 1308.0044 